\title{
Correction: Bhagat, S.; et al. Deep Reinforcement Learning for Soft, Flexible Robots: Brief Review with Impending Challenges. Robotics 2019, 8, 4
}

\author{
Sarthak Bhagat ${ }^{1,2,+(\mathbb{D}, \text { Hritwick Banerjee }}{ }^{1,3,+}+\mathbb{D}$, Zion Tsz Ho Tse ${ }^{4}(\mathbb{C})$ and Hongliang Ren ${ }^{1,3,5, *(\mathbb{D})}$ \\ 1 Department of Biomedical Engineering, Faculty of Engineering, 4 Engineering Drive 3, National University \\ of Singapore, Singapore 117583, Singapore; sarthak16189@iiitd.ac.in (S.B.); banerjee@is.mpg.de (H.B.) \\ 2 Department of Electronics and Communications Engineering, Indraprastha Institute of Information \\ Technology, New Delhi 110020, India \\ 3 Singapore Institute for Neurotechnology (SINAPSE), Centre for Life Sciences, National University of \\ Singapore, Singapore 117456, Singapore \\ 4 School of Electrical \& Computer Engineering, College of Engineering, The University of Georgia, \\ Athens, GA 30602, USA; ziontse@uga.edu \\ 5 National University of Singapore (Suzhou) Research Institute (NUSRI), Suzhou Industrial Park, \\ Suzhou 215123, China \\ * Correspondence: ren@nus.edu.sg; Tel.: +65-6601-2802 \\ + These authors equally contributed towards this manuscript.
}

Received: 9 October 2019; Accepted: 12 October 2019; Published: 28 October 2019

The authors wish to make the following corrections to this paper [1]:

1. In Figure 1 of this paper [1], the caption was revised with the permission from the publishers as "Various applications of SoRo. Reprinted (adapted) with permission from [20-22]. Copyright 2017, Elsevier B.V. Copyright 2016, American Association for the Advancement of Science. Copyright 2017, National Academy of Sciences."

2. In Table 2 of this paper [1], the caption was revised with the permission from the publishers as "SoRo applied to achieve state-of-the-art results alongside sub-domains where its utilization with deep reinforcement learning (DRL) and imitation learning techniques presently occur. Pictures adapted with permission from [40,41]. Copyright 2014, Mary Ann Liebert, Inc., publishers. Copyright 2017, American Association for the Advancement of Science."

3. In Figure 2 of this paper [1], the caption was revised with the permission from the publishers as "Training architecture of a Deep Q-Network (DQN) agent. Picture adapted with permission from [47]. Copyright 2018, American Association for the Advancement of Science."

4. In Figure 3 of this paper [1], the caption was revised with the permission from the publishers as “Training architecture of a Deep Deterministic Policy Gradients (DDPG) agent. The blue lines portray the updated equations. Picture adapted with permission from [47]. Copyright 2018, American Association for the Advancement of Science."

5. In Figure 5 of this paper [1], the caption was revised with the permission from the publishers as "Expected application of DRL techniques in the task of navigation. Inset adapted with permission from [72]. Copyright 2018, American Association for the Advancement of Science."

6. In Figure 7 of this paper [1], the caption was revised with the permission from the publishers as "Soft Robot Simulation on SOFA using Soft-robotics toolkit. Figure adapted with permission from [137]. Copyright 2017, IEEE."

7. In Figure 8 of this paper [1], the caption was revised with the permission from the publishers as "Training Architecture of CycleGAN and CyCADA [92,138]. Figure adapted with permission from [139]. Copyright 2018, Mary Ann Liebert, Inc., publishers." 
8. In Table 4 of this paper [1], the caption was revised with the permission from the publishers as, "Instances of bio-inspired soft robotics applications that make use DRL or Imitation Learning technologies. Picture adapted with permission from [47,162-164]. Copyright 2018, American Association for the Advancement of Science. Copyright 2016, Springer Nature Limited. Copyright 2011, IEEE. Copyright 2016, John Wiley Sons, Inc."

The changes do not affect the scientific results. The manuscript will be updated and the original will remain online on the article webpage, with a reference to this Correction. The authors would like to apologize for any inconvenience caused to the readers by these changes.

\section{References}

1. Bhagat, S.; Banerjee, H.; Ho Tse, Z.T.; Ren, H. Deep Reinforcement Learning for Soft, Flexible Robots: Brief Review with Impending Challenges. Robotics 2019, 8, 4. [CrossRef]

(C) 2019 by the authors. Licensee MDPI, Basel, Switzerland. This article is an open access article distributed under the terms and conditions of the Creative Commons Attribution (CC BY) license (http://creativecommons.org/licenses/by/4.0/). 